\title{
Energy consumption, cultural background and payment structure
}

\author{
Alexander Beunder, Loek Groot* \\ Utrecht University School of Economics (U.S.E.), Utrecht University, PO Box 80125, 3508 TC Utrecht, The Netherlands
}

\section{A R T I C L E I N F O}

\section{Article history:}

Received 22 May 2014

Received in revised form

19 December 2014

Accepted 27 January 2015

Available online 7 February 2015

\section{Keywords:}

Energy consumption

Incentives

Energy conservation

Crowding theory

Cultural background

Culture

\begin{abstract}
A B S T R A C T
In order to reduce the ecological footprint of households and mitigate anthropogenic climate change, policy makers need to understand which incentives drive household energy consumption. Economists tend to rely solely on financial instruments, but these might have unintended consequences on energy consumption through 'non-economic' channels. According to what we call the Hackett-Lutzenhiser hypothesis, the relation between households' cultural background and their energy consumption differs under different payment structures. The electricity consumption of households in the Netherlands is categorized according to two different payment structures: unit metered, where households pay for their private electricity consumption and master metered, where electricity costs are lump sum included in the rent. Our findings show that the range of variation in electricity consumption across cultural backgrounds is lower among unit metered than among master metered households, in line with the HackettLutzenhiser hypothesis. The policy implication is that consumers' preferences cannot be simply taken as given, as is customary in standard economic models, but interact with the structure of financial incentives. Taxes and subsidies, or fixed and flexible rates in energy bills, not only change relative prices but may also interact with people's preferences.
\end{abstract}

๑) 2015 Elsevier Ltd. All rights reserved.

\section{Introduction}

To design policies which aim to more environmental-friendly behavior of households, policy makers need to understand which incentives will reduce households' energy consumption (Benabou and Tirole 2006; Galarraga et al., 2011 provides an overview of the energy consumption literature). Economists tend to focus on financial incentives such as taxes and subsidies which change relative prices, e.g. a carbon tax raises the relative price of carbonintensive goods and a subsidy for solar panels reduces the price of clean electricity. However, to fully understand the effect of financial incentives on households' energy consumption, non-economic drivers are also relevant (Bartiaux, 2007; Green, 2004; Lutzenhiser, 1993).

Firstly, because the significance of non-economic factors psychological, social and cultural - in household energy consumption has been established in various studies (Stern, 1999;

\footnotetext{
* Corresponding author. Visiting address: Kriekenpitplein 21-22, 3584 EC Utrecht, The Netherlands. Tel.: +31 (0)302539731.

E-mail address: L.F.M.Groot@uu.nl (L. Groot).
}

Sütterlin et al., 2011; Wilhite et al., 1996). McMakin et al., 2002 document research that show that economic factors do not fully explain energy use behavior as, for instance, consumers may ignore strong financial incentives to conserve energy, while other consumers continue to conserve energy even after the financial incentive was abolished or reduced. Desmedt et al. (2013) report about a Belgian project to influence household energy consumption by different tools, among which an energy diary, an extensive energy advice informing the household on potential savings in heating and electricity and an electrical audit, limited to electrical appliances only. One of the conclusions of their study (Desmedt et al., 2013, 462) is that "households decide on interventions to save energy in similar ways as they decide on other types of consumption. The consumption has to express a certain message. This means that invisible interventions like wall insulation or boiler replacement are seriously put at a disadvantage". By installing solar panels or a solar boiler on the roof, one can signal to the world that one is concerned with the environment, but this signal is not produced with interventions that are invisible, although their energy saving potential may be far higher. Moreover, a household may use its financial savings from an intervention which is visible to the 
social environment, like solar panels, to consume more energy through less visible kinds of energy consumption, like flying to more distant destinations for holidays. This 'rebound effect' is related to the Jevons Paradox, which states that improvements in energy efficiency can even lead to higher total energy consumption (Polimeni et al., 2008). Although the Jevons Paradox is based on a micro-economic argument, it may be deeply intertwined with social contexts which, according to Desmedt et al., 2013, define in which (visible) areas households feel socially compelled to save energy and in which (invisible) areas they feel less constrained to consume energy. In short, it seems to make sense to place "consumption in a broader and more social context than that of the individual 'decision maker'" (Hackett and Lutzenhiser, 1991: 466).

Secondly, because the financial incentives designed to change energy consumption patterns may have unintended consequences through these non-economic channels (Bowles, 2008; Dolan and Metcalfe, 2013; Gneezy et al., 2011). Frey and Jegen (1999, 2001) provide a long list of empirical examples of crowding effects where monetary incentives undermine (crowding-out) or strengthen (crowding-in) intrinsic motivations in order to show that the interaction between economic and non-economic drives is empirically relevant rather than just a theoretical possibility. Perhaps the most famous crowding effects are those where financial rewards for volunteers actually reduce their efforts (Halvorsen, 2010), like the claim of Titmuss that a voluntary system for blood donation gives better outcomes than a system of providing financial rewards (Titmuss, 1970) and that the introduction of a monetary fine increased the number of parents who came too late to pick up their children in a daycare centre in Israel (Gneezy et al., 2011).

The overarching hypothesis of this paper is that the strength of non-economic drivers of households energy consumption differ under different financial incentives or payment structures. More specifically, the hypothesis tested is that the introduction of a price mechanism, which provides a direct financial reward for household energy saving, simultaneously weakens the correlation between the cultural backgrounds of households and their energy consumption. This is what we call the Hackett-Lutzenhiser hypothesis, named after an article by the sociologists Bruce Hackett and Loren Lutzenhiser (1991, hereafter abbreviated as HL) who observed this effect in a California apartment complex. When the culturally diverse residents changed from 'master metering' (in which the electricity costs are included as a fixed cost in the rent) to 'unit metering' (in which each household pays an energy bill for its own electricity consumption, that is tenants are billed for individual measured energy usage), the explanatory power of the residents' "culture of origin" on their electricity consumption became significantly smaller. Under master metering, there was a significant correlation between the level of electricity consumption and the residents' cultural background, while after the transition to unit metering, Latin Americans, Asians and Europeans all started to consume relatively equal levels of electricity on average. Electricity consumption became socially 'neutral' according to $\mathrm{HL}$ - neutralizing the role of cultural background in electricity consumption. To use the idiom of crowding theory: the price mechanism seemed to 'crowd out' the influence of cultural background on energy consumption.

To test whether this neutralizing effect of unit metering also applies to Dutch households, we will analyze the relation between cultural background (hereafter abbreviated as background) and electricity consumption for unit metered and master metered households using a cross-sectional dataset from WoON (2012). WoON (2012) is a large government sponsored project in which a large number of characteristics from around 69 thousand Dutch households are collected every three to four years, including data on electricity, gas and water consumption. This dataset provides information on Dutch households' electricity consumption and their backgrounds in three categories (native Dutch, non-Western immigrants and Western immigrants). The results only provide weak evidence for the hypothesis that unit metering weakens the relation between background and energy consumption.

For policy makers, the HL hypothesis is of practical use in the design of energy conservation policies. Policy makers may for instance realize that among master metered households, cultural habits which are wasteful in terms of energy consumption can be combatted by introducing a financial incentive to save energy (unit metering). At the same time, policy makers should realize that a price mechanism comes at the cost of eliminating culturally shaped 'good' habits in terms of energy behavior, because a price mechanism may "suppress social identities" by instilling an "economizing behavior" in consumers, as HL (1991) assert. Simultaneously, the HL hypothesis suggests that even among master metered households with no financial incentive to save energy, there is scope for policy makers to influence energy consumption through a 'cultural channel' as there is a relatively strong relation between background and energy consumption among these households. Policy makers could look for ways to use this correlation among master metered households for a cause, for instance by influencing households' cultural habits directly in order to limit their energy consumption indirectly.

A multidisciplinary approach to study energy consumption, combining insights from economics and sociology, is adopted in order to find out the relation between energy consumption on the one hand and cultural background and payment method on the other hand. This paper is structured as follows. Section 2 provides the reasoning behind the HL hypothesis and summarizes the research method used by HL. Section 3 explains our data and method, highlighting the differences with HL and its implications. Section 4 presents and discusses the results. Section 5 summarizes and concludes.

\section{The Hackett-Lutzenhiser hypothesis}

"[S]eemingly innocent variations in modes of payment for resources consumed, or payment structures, are in fact social structures - so that changes in them involve changes in status relations and hence implicate consumption patterns." - HL (1991: 466)

HL (1991) researched the effects of a change in billing method in a culturally diverse California apartment complex, inhabited by Asians, Europeans, Latin Americans, Africans and tenants from the Middle East. Overnight and for all apartments simultaneously, the billing method was changed during the summer of 1985 from master metering with energy costs included in the housing rent to unit metering in which each household was billed for their individual energy consumption. Using panel data of residents' electricity consumption, they observed a large drop in electricity consumption among all income classes and across all backgrounds. ${ }^{1}$ For our purposes even more salient is their finding,

\footnotetext{
${ }^{1}$ Combining their data research with field observations, HL (1991: 457) noticed how virtually all income classes "simply turned off their air conditioners" after the change to unit metering, which could not be explained by income and price variables. This act of turning off air conditioners "brought almost everyone to the same consumption level". They argued that "aggregated (and perhaps preset or "fixed") costs are intrinsically less painful than disaggregated or variable costs, even where the totals involved are the same. A more social version of this argument would hold that a shared cost is easier to assume than a personal or private cost of the same amount" (HL, 1991: 461).
} 


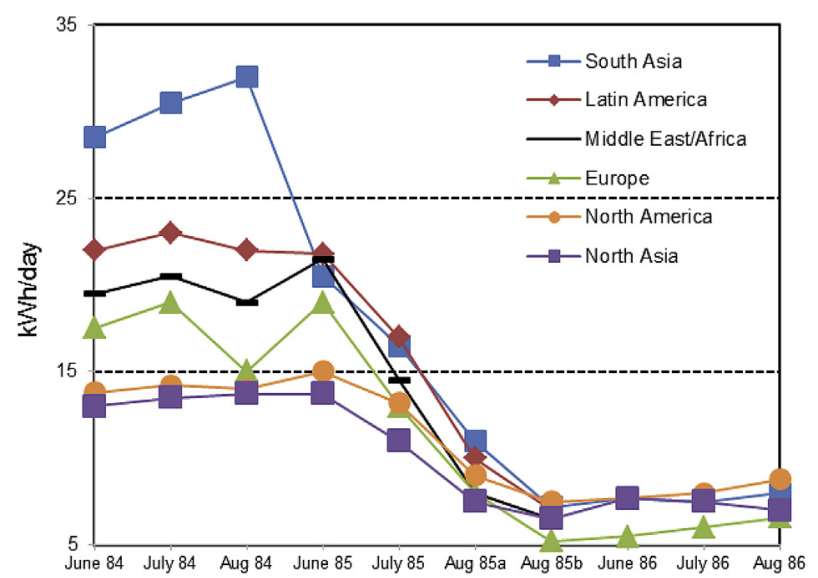

Fig. 1. Billing conversion and cultural variations in summer electricity consumption $(\mathrm{n}=243)$. Source: Reproduced from Hackett and Lutzenhiser, 1991: 463.

illustrated in Fig. 1, that while under master metering there was a significant relation between households' background and electricity consumption, under unit metering this relation disappeared. ${ }^{2}$ As HL (1991) note, their graph does not correct for any correlation between background and income and may therefore give the false impression that energy consumption mainly depends on background, while it actually also depends on income. However, HL (1991: 463) state that "in this sample at least, [nationality and income, while not independent], are not related to energy consumption in a straightforward way", that is, they do not observe a relation between income and consumption levels in their sample which would fit squarely with micro-economic predictions. Regressions with the control variables income and households size confirmed that under unit metering the influence of background on electricity consumption diminished: under master metering, four out of the five background variables were significant at the five percent level, while under unit metering only two were significant.

To explain these results, HL (1991: 467) argue that people from different countries “... bring with them habits of living that may, as we have seen, influence their use of the technologies now available to them". This cultural effect can explain why electricity consumption differs before the introduction of unit metering. Their sociological explanation for the diminished relation between background and electricity consumption is that billing methods "confer and suppress social identities", where unit metering changed people's social status from being 'consumers' enjoying the "privileges of membership" - to 'customers'. Residents as customers started to show "what might be termed 'economizing' behavior" (HL, 1991: 462) in which the role of background was apparently much smaller. As the right side of Fig. 1 shows, energy consumption became "socially 'neutral'” (HL, 1991: 464).

Another way to look at this is to acknowledge that markets, instead of being influenced by cultures - the 'markets have cultures' view, in the words of Levin (2008) - can also be considered as cultures themselves - the 'markets are cultures' view. According to the latter view, markets only function to their full potential when a market culture is created: "The 'markets are culture' or constitutive approach takes as its central claim that market actors, objects, and activities must be made stable before they can look and act like economic markets. [ ... ] The world is not populated with buyers and sellers, commodities, rational agency, and market exchange. These have to be created cognitively, structurally and legally" (Levin, 2008:7). From this perspective, unit metering creates a market culture in residents' energy consumption behavior, commodifying something that was free before and instilling a calculative agency which is itself "a deeply cultural convention", where calculative agency is defined by Levin as "the assumption that individuals assess exchange options against a set of personal preferences and make decisions based on these preferences" (Levin, 2008: 8). If a market is considered a culture itself, then it is only logical that the introduction of a market culture diminishes the role of culture or background previously driving energy consumption decisions. While under master metering it may be easier to freely consume electricity according to culturally shaped habits of living, under unit metering there is a financial incentive to take into account which may dominate, conflict with or even eliminate these cultural habits.

The suppression of background under unit metering or any other price mechanism does not imply that consumers neatly act as rational individual decision makers independent from social influences, as even under unit metering HL (1991: 458) "found little evidence - formal or informal - of the calculation of energy costs and benefits that one might predict from a strictly economic model of consumption". What's more, in their survey HL observed support for their argument that "energy use should properly be seen as governed by considerations of appropriateness or "normality" in given social settings or situations", even under unit metering (HL, 1991: 462). This argument is supported by more recent findings of Schultz et al. (2007), who discovered that unit metered households use perceived social averages as guidelines in their own consumption decisions. Schultz et al. (2007) found in a field experiment on energy conservation in which households were informed about average neighborhood energy usage, that those who were below the average increased their energy consumption, while those who were above the average decreased their energy consumption. This gravitation effect toward the mean occurred because "individuals use their perceptions of peer norms as a standard against which to compare their own behavior" (Schultz et al., 2007: 1). ${ }^{3}$

Our HL hypothesis, then, does not state that unit metering 'liberates' households from their culturally and socially shaped habits and converts them into autonomous, economically rational decision makers, but should rather be interpreted as a prediction of which (perceived) social norms drives consumption decisions under which payment structures. Under master metering, households' cultural backgrounds is expected to drive energy consumption more than under unit metering, while under unit metering other peer norms - which lie beyond the scope of this paper - is expected to drive consumption decisions more than cultural backgrounds.

\footnotetext{
2 As HL (1991) note, their graph does not correct for any correlation between background and income and may therefore give the false impression that energy consumption depends on background, while it actually depends on income. However, as HL (1991) say, "in this sample at least, [nationality and income, while not independent], are not related to energy consumption in a straightforward way" (HL, 1991: 463) as they do not observe a relation between income and consumption levels in their sample which would fit nicely with micro-economic predictions.
}

\footnotetext{
3 The necessary condition to make people gravitate towards this average is correct information about the social average, as people do not automatically gravitate towards the social average but to the perceived social average which can be an incorrect estimate, as Schultz et al. (2007) argue: "The majority of people overestimate the prevalence of many undesirable behaviors, such as alcohol use among peers".
} 
In short, according to the Hacket-Lutzenhiser hypothesis, we expect that the influence of households' background on electricity consumption is smaller under unit metering than under master metering. To our knowledge, there has not been any follow-up research on the influence of households' background on electricity consumption using the comparison between master and unit metered households.
Our model estimates the relation between electricity consumption as a dependent variable and households' background as an explanatory variable for both master metered and unit metered households separately. Control variables are included like households' income levels, educational level, household type and the surface of the rental apartment in square meters. Dummies for households' main source of income are included, as this variable may indicate different lifestyles and hours spent inside the house which could influence electricity consumption significantly. The equation to be estimated is:

$$
\begin{aligned}
\text { Log Electricity }= & \beta_{1}+\beta_{2} \text { Log Income }+\beta_{3} \text { Welfare }+\beta_{4} \text { Pension }+\beta_{5} \text { Student }+\beta_{6} \text { Higher Educated }+\beta_{7} \text { Couple } \\
& +\beta_{8} \text { Couple with child }(\text { ren })+\beta_{9} \text { Single }- \text { parent family with child }(\text { ren })+\beta_{10} \text { Non }- \text { family household } \\
& +\beta_{11} \text { Surface } 70-119 \text { metre }{ }^{2}+\beta_{12} \text { Surface } 120-199 \text { metre }^{2}+\beta_{13} \text { Surface more than } 199 \text { metre }{ }^{2}+\beta_{14} \text { Non } \\
& - \text { Western immigrant }+\beta_{15} \text { Western immigrant }+\varepsilon
\end{aligned}
$$

\section{Methods}

To test the HL hypothesis, the electricity consumption levels of Dutch households from the latest release of the WoON survey (2012) are used, irrespective of the electricity being generated by burning fossil fuels such as coal or gaz, solar power or hydroelectric power. Although WoON also provides data on gas and water consumption, to analyze gas consumption properly is very challenging as it is known to depend not only on apartments' energy efficiency levels but also on temperatures in their region as people use more heating when it is cold (Abrahamse and Steg, 2011), on which there is little to no data in the WoON dataset. ${ }^{4}$ Water consumption is simply less relevant in the context of anthropogenic climate change and greenhouse-gas emissions.

WoON consists of a sample of 69,339 Dutch residents, above the age of eighteen, of which 23,073 respondents (33.3 percent) indicated whether their electricity consumption is master metered or unit metered. In total, 10.22 percent of those 23,073 respondents are master metered. Only residents of rental apartments will be included. This restriction to apartments simplifies the analysis, as households living in detached houses probably face very different social contexts compared to households in apartments. Therefore, the role of background and other social factors which are included in the analysis might be very different in detached houses compared to apartments. As 74.7 percent of all master metered households in the dataset live in apartments, even with this restriction there are still enough observations of this category. Finally, to omit distorting outliers, only respondents with a yearly positive disposable income below 100,000 euro are included (one respondent reported an income of minus 1,051,368 euro and there were only seven master metered households who earned more than 100,000 euro.).

\footnotetext{
${ }^{4}$ The WoON dataset has no information on temperatures. However, it does provide limited information on the 'energy-label' of the households, which roughly indicates their level of energy-efficiency, but only for 25.5 percent of all households in the dataset. Restricting the observations to those with an energy-label would greatly reduce the number of observations. The importance of including energyefficiency, especially when analyzing a relation between gas consumption and background, is emphasized by RIGO (2010). Some of the correlations this research discovered between gas consumption and background might be a result of a spurious correlation between background and households' level of energy efficiency. Especially the 'immigrant' category seemed to be overrepresented in houses with low levels of energy efficiency, explaining their higher levels of gas consumption.
}

The dependent variable Log Electricity is the log of electricity consumption measured in kWh consumed in a year. A log-log model to estimate the coefficient of income is used, as the relation between income level and spending on basic utilities is known to be non-linear, with the share of spending on basic utilities such as electricity to decline with higher income levels (Heffetz, 2011). Welfare, Pension and Student are dummies for households' main source of income, using the salaried workers and self-employed as the reference group. Welfare equals 1 when households' main source of income consists of welfare benefits, Pension equals 1 when a pension is the main source of income and Student equals 1 when the main source of income is student finance. Higher Educated is a dummy which equals 1 if either the respondent or his/ her partner is higher educated (having graduated from a polytechnic school or a university). Dummies for different household types are included with the single person household as the reference group. Surface dummies are includes for different sizes of houses in square metres (using the notation metre ${ }^{2}$ ), with houses below $50 \mathrm{~m}^{2}$ as the reference group.

To test the HL hypothesis - the influence of households' background on their electricity consumption is smaller under unit metering than under master metering - dummies for the background of households are included based on three categories of respondents' geographic origins provided in the WoON dataset; one for non-Western immigrant and one for Western immigrant, with the native Dutch as the reference category. The model will then be estimated for the two types of households (unit metered and master metered) separately, in order to compare the coefficients of the background dummies. If the HL hypothesis holds, the explanatory power of background for electricity consumption will be smaller and/or less significant among the unit metered households than among the master metered households. The error term is represented by $\varepsilon$ and Ordinary Least Squares (OLS) regression with robust standard errors is used to correct for any observed heteroskedasticity.

A few caveats have to be addressed before the results are presented. Firstly, a self-selection bias may occur when people who prefer to consume much electricity and have lower than average environmental concerns choose to live in master metered houses instead of unit metered houses to benefit from seemingly 'free' energy. If people from one of our three background categories are more inclined to choose master metered houses than people from another background for this reason, this may bias our results. 
However, in the Dutch case, long waiting lists exist for rental apartments which are not in the private sector which limits households' choices in terms of housing, and only a small share of the rental apartments from our sample are in the private sector. Moreover, spending on energy is a relatively small part of households' total expenditures and should therefore play a minor role in the choice of housing. Therefore, we do not expect that a selfselection bias is prevalent.

Secondly, we do not control for the devices owned by the household (e.g., types of TV and computer monitors, age of freezer, pumps, etc.), nor for "the amount of time family members spend in the home versus other places (at work, traveling, second home, etc.)" which Thogersen and Gronhoj (2010) point out as important factors influencing electricity consumption. Instead, the income variable will by and large capture the quantity of devices owned by households, as higher income households own more electricity consuming durable goods than lower income households (CBS, 2010). Moreover, the dummies for the main source of households' income might capture some of the variance of time spent inside the home.

Thirdly, the background of the households' respondent may not adequately represent the household in its entirety as other household members may have different backgrounds. For that reason, households with 'mixed couples' - respondents who live with partners of another origin than that of themselves - are excluded, which decreases our number of observations with 6.3 percent.

Fourthly, we have assumed until now that master metered households have no financial incentive to save energy. However, they might have a small financial incentive to save energy, as some master metered apartments receive an annual reimbursement of energy costs when their joint consumption has been below a certain threshold. This reimbursement is generally small and only weakly related to private energy consumption. For simplicity it is assumed that the prospect of a small reimbursement depending on the joint energy use in an apartment complex is not a significant driver of electricity consumption.

Finally, we do not take into account any possible 'rebound effect' through indirect energy consumption. As Abrahamse and Steg (2009) emphasize, private households use energy both directly (gas, electricity, fuel) and indirectly (embedded in the production, consumption and disposal of goods). Whether some households consume less energy in terms of electricity in fact consume much more total electricity through indirect consumption is a question which lies beyond the scope of this paper.

\subsection{Differences with Hackett and Lutzenhiser}

Before presenting our results and comparing them with those of HL (1991), note that there are both similarities and differences between their and our regression.

The similarity is that both methods compare the role of background under two different payment structures, unit metering and master metering. The most important difference is that HL (1991) use panel data, while here cross-sectional data are used. The figure of HL (1991) reproduced in Section 2 above depicts energy consumption over time of the same group of tenants who change overnight from one payment method to another. Here the energy consumption of tenants under different payment methods in 2012 are compared. This complicates a direct comparison, especially because the time period covered by HL (1991) is rather short as their "data included apartment-level meter readings $(n=476$ units) for a one-year period prior to, and one year following, the conversion" from master to unit metering. Their unit metered households have had only one year experience with this individual billing, while the unit metered households in our WoON dataset have had, on average, many more years of experience with individual billing. Our results may therefore differ if, for instance, the diminished role of background after the conversion to unit metering, which HL (1991) observe, would in fact re-emerge over the years. Therefore, our model should not be seen as a replication of HL (1991) with simply another dataset, but as a complementary test to see whether the conclusions of HL (1991) also apply to households which have had more time to adapt to their billing methods.

Regarding the variables, both regressions include control variables for income levels, family types and dwellings size, albeit with different categories. However, HL (1991) use some control variables which we omit (length of residence, a dummy for number of hot days and the possession of air conditioners) and we use some control variables absent in HL (1991), in particular main source of income and education level. Finally, we have only three background categories while HL (1991) have six, which could diminish the observed variance in electricity consumption we observe compared to HL (1991). ${ }^{5}$

Regarding the data itself, one difference is that the population in the sample of HL (1991) "was relatively homogeneous in respect to age, education, and incipient social standing" and "included mostly graduate/professional student families" (HL, 1991: 455). In our sample, the population was more heterogeneous and included pensioners, students, salaried workers and business owners, with both high and low educated. The apartments in HL (1991) were also homogeneous as "the apartments are all virtually identical" (HL, 1991: 455), while in our sample the apartments are not identical, although our analysis is restricted to apartments and detached houses are exluded.

Lastly, the data of HL (1991) was "supplemented by a limited amount of participant observation that fell far short of a full-blown ethnography but did aid our interpretive work" (HL, 1991: 450). Though we acknowledge the value of this anthropological research method, we have limited ourselves to a statistical analysis.

\section{Results and discussion}

Table 1 shows the results from the regression testing the HL hypothesis. First of all, it is interesting to see that the income variable is significantly positive for the unit metered households, but not significant for the master metered households. This suggests that energy consumption is considered as more 'free' under the latter, while a certain 'calculative agency' prevails under unit metering. This is in line with the results of HL (1991) in which at least one of two income dummies becomes significant under unit metering, while no income dummy was significant under master metering.

The dummies for main sources of income suggest that, compared to the reference group of salaried workers and selfemployed, pensioners consume less electricity among master metered households and welfare recipients consume more among unit metered households. Note that all the household type dummies, where the single person household is the reference, are statistically significant and have the expected positive sign. All the surface dummies have the expected positive sign, but not all of them are statistically significant. Among the unit metered

\footnotetext{
5 One reason to expect our categories to show smaller differences is that the variance of electricity consumption between different 'non-Western' backgrounds may be high. Note for instance that in Fig. 1, under master metering, South Asians have the highest electricity consumption level while North Asians have the lowest level, and both would be counted as 'non-Western immigrant' if our categories were used.
} 
Table 1

The effect of background on electricity consumption.

\begin{tabular}{lll}
\hline & $\begin{array}{l}\text { Master metered } \\
\text { households } \\
\mathrm{N}=1048\end{array}$ & $\begin{array}{l}\text { Unit metered } \\
\text { households } \\
\mathrm{N}=9924\end{array}$ \\
\hline Log Income & $-0.010(0.30)$ & $0.077(4.60)^{* *}$ \\
Welfare & $-0.082(1.27)$ & $0.034(1.97)^{*}$ \\
Pension & $-0.171(3.80)^{* *}$ & $-0.011(0.80)$ \\
Student & $-0.085(1.37)$ & $0.077(1.46)$ \\
Higher educated & $-0.027(0.63)$ & $-0.065(4.58)^{* *}$ \\
Couple & $0.311(5.92)^{* *}$ & $0.249(15.20)^{* *}$ \\
Couple with child(ren) & $0.363(3.35)^{* *}$ & $0.472(20.74)^{* *}$ \\
Single-parent family & $0.346(2.75)^{* *}$ & $0.327(14.53)^{* *}$ \\
$\quad$ with child(ren) & $0.269(4.70)^{* *}$ & \\
Non-family household & $0.035(0.89)$ & $0.226(6.25)^{* *}$ \\
Surface 70-119 m ${ }^{2}$ & $0.227(3.24)^{* *}$ & $0.077(6.70)^{* *}$ \\
Surface 120-199 m ${ }^{2}$ & $0.187(0.92)$ & $0.109(3.47)^{* *}$ \\
Surface more than 199 $\mathrm{m}^{2}$ & $-0.163(2.95)^{* *}$ & $0.186(2.38)^{*}$ \\
Non-Western immigrant & $0.074(1.26)$ & $-0.073(4.27)^{* *}$ \\
Western immigrant & $7.647(22.92)^{* *}$ & $-0.013(0.61)$ \\
Constant & 0.11 & $6.626(39.87)^{* *}$ \\
R-squared & & 0.10 \\
\hline
\end{tabular}

Robust $\mathrm{t}$ statistics in parentheses.

* significant at $5 \%$; ${ }^{* *}$ significant at $1 \%$.

household, the coefficients for the surface dummies increase as the surface increases.

Background seems to have a larger effect on electricity consumption among households under master metering than under unit metering, as predicted by the HL hypothesis. Based on this logdummy model, the non-Western immigrant consumes 16.3 percent less electricity than the native Dutch under master metering, significant at the one percent level. However, under unit metering the non-Western immigrant consumes only 7.3 percent less, also significant at the one percent level. Although the Western immigrant does not consume significantly more or less electricity compared with the native Dutch reference category, the best estimate is that they consume 7.4 percent more than the reference category among the master metered households. Therefore the variation in electricity consumption categorized by background ranges from -16.3 to +7.4 percent, with the Western immigrant on top, the native-Dutch in the middle and non-Western immigrants at the bottom, which gives a total range of 23.7 percent. Among the unit metered households, the total range in the variation of electricity consumption is only around a third of this: it is reduced to 8.6 percent. However, due to the insignificance of the dummy for Western immigrants, comparing unit metered with master metered households, more importance must be attached to the significantly lower electricity consumption of non-Western immigrants relative to native Dutch households, both in master and unit metered households. Our results therefore deliver a picture which is similar, albeit less pronounced, to Fig. 1 obtained from HL (1991), namely that the range in electricity consumption - where households are identified by background and controlled for other variables - is wider among master than under unit metered households. Admittedly, this higher observed range in electricity consumption across backgrounds can only be considered as weak evidence of the HL hypothesis because a standard Student t-test on the difference between the background dummy coefficients reveals that they are not significantly different (e.g. the hypothesis that the coefficients for the non-Western immigrants under master and under unit metered are equal cannot be rejected). Thus, although the influence of background on electricity consumption has not been totally eliminated or 'crowded-out' under unit metering, the differences between the three backgrounds categories have become smaller, in line with the hypothesis of $\mathrm{HL}$ that electricity consumption levels tend to converge when households have to pay for their own private electricity consumption.

\section{Conclusions}

In this article a multidisciplinary approach to study households' energy consumption, combining insights from economics and sociology, is adopted. The starting point was the Hackett and Lutzenhiser (1991) observation that unit metering (billing households for their private energy consumption) weakens the relation between cultural background and energy consumption. Using a large sample of Dutch households, our results provide only weak support for the Hacket-Lutzenhiser hypothesis in so far that the observed range in electricity consumption among households with different cultural backgrounds is more compressed under unit metering compared to master metering, at least for the sample of rental apartment households used in this study. Using a logdummy model with electricity consumption as the dependent variable and controlling for income, source of income, education level, type of household and surface area of the dwelling, nonWestern immigrants consume approximately 16 percent less electricity than the native Dutch under master metering, compared to approximately 7 percent under unit metering. As Hackett and Lutzenhiser (1991) suggest, a transition from master to unit metering instills a market culture and "economizing behaviour" with the effect of neutralizing the influence of households' cultural backgrounds.

However, standard Student t-tests show that although the range narrows, it cannot be concluded that the background coefficients under master and unit metering are significantly different. This may indicate that the role of background under unit metered households re-emerges over time, as the households in our crosssectional sample have lived more time on average under unit metering than the households in the panel dataset of HL (1991) which have only one year of experience with this billing method. It may also indicate that our small number of background categories hides a larger 'true' variance between a more detailed set of background categories. The results only apply to The Netherlands and might differ for other countries, with more profound cleavages in cultural backgrounds of their populations.

If the HL hypothesis holds, then the policy implication is that consumers' preferences cannot be simply taken as given, as is customary in standard economic models, but interact with the structure of financial incentives. As HL (1991: 466) argue, we should place "consumption in a broader and more social context than that of the individual 'decision maker"'. If so, it has implications for both the scope and effectiveness of (environmental) policies. Economists tend to constrain themselves to the price instrument, that is, using price elasticities in order to calculate the optimal tax and subsidy levels. However, taxes and subsidies, or fixed and flexible rates in energy bills, not only change relative prices but may also interact with people's preferences. This complicates the identification of the optimal policy, but also widens the range of policy options. Avenues for further research are to test whether results supporting the Hackett- Lutzenhiser hypothesis are robust or not, for instance by using datasets of different countries, or by focusing on different consumption goods.

\section{References}

Abrahamse, W., Steg, L., 2009. How do socio-demographic and psychological factors relate to households' direct and indirect energy use and savings? J. Econ. Psychol. 30, 711-720.

Abrahamse, W., Steg, L., 2011. Factors related to household energy use and intention to reduce it: the role of psychological and socio-demographic variables. Hum. Ecol. Rev. 18, 30-40.

Bartiaux, F., 2007. Does environmental information overcome practice compartmentalisation and change consumers' behaviours? J. Clean. Prod. 16, 1170-1180.

Benabou, R., Tirole, J., 2006. Incentives and prosocial behavior. Am. Econ. Rev. 96 (5), 1652-1678. 
Bowles, S., 2008. Policies designed for self-interested citizens may undermine "the moral sentiments": evidence from economic experiments. Science 320, 1605-1609.

CBS, 2010. Duurzame Goederen; Bezit Naar Huishoudenskenmerken. April 27, 2010 Available at: http://statline.cbs.nl/StatWeb/publication/?DM=SLNL\&PA= 37926\&D1 $=0-40 \& \mathrm{D} 2=0,8-14,30-31,34-36 \& D 3=1 \& \mathrm{VW}=\mathrm{T} \quad$ (accessed on 17.07.13.).

Desmedt, J., Vekemans, G., Maes, D., 2013. Ensuring effectiveness of information to influence household behavior. J. Clean. Prod. 16, 455-462.

Dolan, P., Metcalfe, R., 2013. Neighbors, Knowledge, and Nuggets: Two Natural Field Experiments on the Role of Incentives on Energy Conservation. CEP Discussion paper No 1222, June, 2013. Available at: http://cep.lse.ac.uk/pubs/download/ dp1222.pdf (accessed on 17.07.13.).

Frey, B.S., Jegen, R., 1999. Motivation Crowding Theory: a Survey of Empirical Evidence. Working Paper Series No.26. Institute of Empirical Research in Economics, University of Zürich.

Frey, B.S., Jegen, R., 2001. Motivation crowding theory. J. Econ. Surv. 15 (5), 589-608.

Galarraga, I., Markandya, A., Eguino, M.G. (Eds.), 2011. Handbook of Sustainable Energy. Edward Elgar Publishing.

Gneezy, U., Meier, S., Rey-Biel, P., 2011. When and why incentives (Do not) work to modify behavior. J. Econ. Perspect. 25 (4), 1-21. Fall 2011.

Green, E., 2004. Explaining cross-National variation in energy consumption. Int. J. Sociol. 34 (1), 9-32. Spring 2004.

Hackett, B., Lutzenhiser, L., 1991. Social structures and economic Conduct: Interpreting variations in household energy consumption. Sociol. Forum 6 449-470.

Halvorsen, B., 2010. Effects of Norms and Policy Incentives on Household Recycling: an International Comparison. Statistics Norway, Research Department. Discussion Papers No. 627, August 2010.

Heffetz, Ori, 2011. A test of conspicuous consumption: visibility and income elasticities. Rev. Econ. Statistics 93, 1101-1117.
Levin, P., 2008. Culture and markets: how economic sociology conceptualizes culture. Ann. Am. Acad. Political Soc. Sci. 619, 114-129. Cultural Sociology and Its Diversity (Sep., 2008).

Lutzenhiser, L., 1993. Social and behavioral aspects of energy use. Annu. Rev. Energy Environ. 18, 247-289.

McMakin, A.H., Malone, E.L., Lundgren, R.E., 2002. Motivating residents to conserve energy without financial incentives. Environ. Behav. 34 (6), 848-864.

Polimeni, John M., Mayumi, Kozo, Giampietro, Mario, Alcott, Blake, 2008. The Jevons Paradox and the Myth of Resource Efficiency Improvements. Earthscan.

RIGO Research en Advies, commissioned by Ministerie van VROM, 2010. Energiegedrag in de woning: Aanknopingspunten voor de vermindering van het energiegebruik in de woningvoorraad. Available at: http://www.rijksoverheid. nl/documenten-en-publicaties/rapporten/2010/03/11/energiegedrag-in-dewoning.html (accessed on 17.07.13.).

Schultz, P.W., Nolan, J.M., Cialdini, R.B., Goldstein, N.J., Griskevicius, V., 2007. The constructive, destructive, and reconstructive power of social norms. Psychol. Sci. 18 (5), 429-434.

Stern, P., 1999. Information, incentives, and proenvironmental consumer behavior. J. Consum. Policy 22, 461-478.

Sütterlin, B., Brunner, T.A., Siegrist, M., 2011. Who puts the most energy into energy conservation? A segmentation of energy consumers based on energy-related behavioral characteristics. Energy Policy 39 (12), 8137-8152.

Thogersen, J., Gronhoj, A., 2010. Electricity saving in households-A social cognitive approach. Energy Policy 38 (12), 7732-7743.

Titmuss, Richard M., 1970. The Gift Relationship. Allen and Unwin.

Wilhite, H., Nakagame, H., Masuda, T., Yamaga, Y., Haneda, H., 1996. A cross-cultural analysis of household energy-use behaviour in Japan and Norway. Energy Policy 24 (9), 795-803.

WoON, 2012. WoON2012: release 1.0-WoonOnderzoek Nederland 2012 (voor overheid, universiteiten en overige partijen). Dataset developed by BZK in collaboration with CBS. Available at DANS. https://www.easy.dans.knaw.nl/ui/home. 